\title{
Existence and Exponential Convergence of almost Periodic Solutions for a Discrete Nicholson's Blowflies Model with Nonlinear Harvesting Term
}

\author{
Jehad O. Alzabut* \\ Department of Mathematics and Physical Sciences, Prince Sultan University, P. O. Box 66833, 11586 Riyadh, Saudi Arabia
}

Received: 4 Mar. 2013, Revised: 6 Jun. 2013, Accepted: 8 Jun. 2013

Published online: 1 Sep. 2013

\begin{abstract}
We establish sufficient conditions for the existence and exponential convergence of positive almost periodic solutions for a discrete Nicholson's blowflies model with nonlinear harvesting term. A result concerning the persistence of the solutions is provided prior to proving the main theorems. The validity of the main results is demonstrated by a numerical example.
\end{abstract}

Keywords: Almost periodic solution; Exponential stability; Discrete Nicholson's blowflies model; Nonlinear harvesting term.

\section{Introduction}

One of the most popular population models is the well known Nicholson's blowflies model

$$
x^{\prime}(t)=-\alpha x(t)+\beta x(t-\tau) e^{-\gamma x(t-\tau)},
$$

which was proposed by Gurney et al. in [1] to describe the population of the Australian sheep-blowfly and to agree with Nicholson's experimental data [2]. Here $x(t)$ is the size of the population at time $t, \beta$ is the maximum per capita daily egg production, $1 / \gamma$ is the size at which the blowfly population reproduces at its maximum rate, $\alpha$ is the pair capita daily adult death rate and $\tau$ is the generation time. The dynamical behavior of solutions for various modifications of this model and its discrete analogue has been extensively studied by many authors during the last couple of decades. For the background of model (1), we suggest the reader to consult some relevant papers $[3,4,5,6,7,8,9,10,11]$.

It is well-known that the optimal management of renewable resources has direct relationship to sustainable development of population. One way to handle this is to study population models subject to harvesting, dispersal or competition. Biologists have purported that the process of harvesting of population species, in particular, is of great significance in exploitation of biological resources such as in fishery, forestry and wildlife management. Assuming that the harvesting is a function of the delayed estimate of the true population, the authors in [12] have put forward a question about the asymptotic behavior of Nicholson's model containing linear harvesting term of the form

$$
x^{\prime}(t)=-\alpha x(t)+\beta x(t-\tau) e^{-\gamma x(t-\tau)}-H x(t-\sigma),
$$

where $\alpha, \beta, \tau, \gamma, \sigma, H \in(0, \infty)$. In response to the raised question, there have appeared many results concerning the investigation of periodic and almost periodic behaviors of equation (2) via employing several utilities such as fixed point theorems and coincidence degree theory $[13,14,15$, $16,17,18]$. The generalized Nicholson's blowflies model with a nonlinear harvesting term of the form

$$
\begin{aligned}
x^{\prime}(t) & =-\alpha(t) x(t)+\sum_{k=1}^{N} \beta_{k}(t) x\left(t-\tau_{k}(t)\right) e^{-x\left(t-\tau_{k}(t)\right)} \\
& -H(t, x(t))
\end{aligned}
$$

has been recently attacked in the new paper [19]. Indeed, the standard Leray-Schauder degree techniques are used to establish necessary and sufficient conditions for the existence of at least one $T$-periodic solutions.

Besides their theoretical significance, difference equations with almost periodic coefficients have shown important influence in mathematical biology. A model governed by these types of equations could be used to mimic a population's response to seasonal fluctuations in its environment; see for instance the remarkable

\footnotetext{
${ }^{*}$ Corresponding author e-mail: jalzabut@psu.edu.sa
} 
monographs [20,21,22]. Motivated by the above justifications, numerous number of papers studying population models described by difference equations have been published by many researches over the last three decades. Following this trend, the investigation of the almost periodic solutions for the discrete counterpart of equation (2) has been recently investigated by Alzabut et al. in [23]. In this paper, however, we shall consider the discrete Nicholson's blowflies model with nonlinear harvesting term of the form

$$
\begin{aligned}
\Delta x(n) & =-\alpha(n) x(n+1)+\beta(n) x(n-\tau(n)) e^{-\gamma(n) x(n-\tau(n))} \\
& -H(n, x(n-\sigma(n))),
\end{aligned}
$$

where $n \in \mathbb{Z}$ and $\alpha, \beta, \gamma, \tau, \sigma: \mathbb{Z} \rightarrow[0, \infty)$ and $H: \mathbb{Z} \times \mathbb{Z}^{m} \rightarrow[0, \infty)$. We shall employ the contraction mapping principle to establish sufficient conditions for the existence of an almost periodic solution for (4). By constructing an appropriate Lyapunov functional, we also prove that the solutions of (4) converge exponentially to an almost periodic solution. We provide a numerical example to illustrate the effectiveness of the main theorems. To the best of author's observation, no paper has been published regarding the investigation of almost periodicity of equation (4). Comparing with some earlier works, the result of this paper is novel and presents a different approach.

\section{Preliminary Assertions}

Let $\mathbb{Z}$ and $\mathbb{Z}^{m}$ be the sets of integer numbers and the $m$-dimensional integer vectors, respectively. For a bounded sequences $g$ and $f$ defined on $\mathbb{Z}$ and $\mathbb{Z} \times \mathbb{Z}^{m}$, respectively, we define $g^{+}, g^{-}, f^{+}$and $f^{-}$as follows $g^{+}=\sup _{n \in \mathbb{Z}} g(n), \quad g^{-}=\inf _{n \in \mathbb{Z}} g(n) \quad$ and $f^{+}=\sup _{(n, x) \rightarrow\left(\mathbb{Z}, \mathbb{Z}^{m}\right)} f(n, x), f^{-}=\inf _{(n, x) \rightarrow\left(\mathbb{Z}, \mathbb{Z}^{m}\right)} f(n, x)$. In the sequel, we assume that

$$
\alpha^{-}>0, \beta^{-}>0, \gamma^{-}>0
$$

and

$$
r=\max \left\{\tau^{+}, \sigma^{+}\right\}>0 .
$$

Let $I=\{-r,-r+1, \ldots,-1,0\}, C=\{\varphi: I \rightarrow \mathbb{Z}\}$ and $C^{+}=$ $\{\varphi \in C: \varphi \geq 0, \varphi(0)>0\}$. For each $\varphi \in C$, we define the norm of $\varphi$ as $\|\varphi\|_{0}=\max _{s \in I}|\varphi|$. Denote $x_{n}=x(n+s)$ for all $s \in I$. For any $\varphi \in C^{+}$, it is easy to see that there is a unique solution $x(n, 0, \varphi)$ of (4) with

$$
x_{0}=\varphi
$$

and $x(n, 0, \varphi)>0$ for all $n \in \mathbb{Z}$.

Definition 1.[24] A sequence $x: \mathbb{Z} \rightarrow \mathbb{R}^{m}$ is said to be almost periodic sequence if the $\varepsilon$-translation set of $x$ :

$$
E\{\varepsilon, x\}:=\{\tau \in \mathbb{Z}:|x(n+\tau)-x(n)|<\varepsilon\},
$$

for all $n \in \mathbb{Z}$ is a relatively dense set in $\mathbb{Z}$ for all $\varepsilon>0$, that $i s$, for any given $\varepsilon>0$, there exists an integer $l>0$ such that each discrete interval of length $l$ contains an integer $\tau=\tau(\varepsilon) \in E\{\varepsilon, x\}$ such that

$$
|x(n+\tau)-x(n)|<\varepsilon,
$$

for all $n \in \mathbb{Z}, \tau$ is called the $\varepsilon$-translation number of $x(n)$.

Definition 2.[24] Let $f: \mathbb{Z} \times D \rightarrow \mathbb{R}^{m}$, where $D$ is an open set in $\mathbb{R}^{m}, f(n, x)$ is said to be almost periodic in $n$ uniformly for $x \in D$ if for any $\varepsilon>0$ and any compact set $S$ in $D$, there exists a positive integer $l(\varepsilon, S)$ such that any interval of length $l(\varepsilon, S)$ contains an integer $\tau$ for which

$$
|f(n+\tau, x)-f(n, x)|<\varepsilon,
$$

for all $n \in \mathbb{Z}$ and $x \in S, \tau$ is called the $\varepsilon$-translation number of $f(n, x)$.

We consider equation (4) under the assumptions:

H. $1 \alpha, \beta, \gamma, \tau, \sigma: \mathbb{Z} \rightarrow[0, \infty)$ are almost periodic sequences;

H.2H: $\mathbb{Z} \times \mathbb{Z}^{m} \rightarrow[0, \infty)$ is almost periodic sequence and there exists $L_{H}>0$ such that

$$
\|H(n, x)-H(n, y)\| \leq L_{H}\|x-y\| .
$$

Further, we assume that

A.1There exist two constants $\Gamma_{1}$ and $\Gamma_{2}$ such that

$$
\left(\frac{\beta}{\gamma}\right)^{+} \frac{1}{\alpha^{-} e}<\Gamma_{1}, \frac{\beta^{-}}{\alpha^{+}} \Gamma_{1} e^{-\gamma^{+} \Gamma_{1}}-\frac{H^{+}}{\alpha^{+}}>\Gamma_{2}
$$

and $\Gamma_{1}>\Gamma_{2}$.

The following result tells that every solution of (4) persists.

Lemma 1.Let A.1 hold. Then for $\varphi \in C^{0}:=\left\{\varphi: \Gamma_{2}<\varphi(n)<\Gamma_{1}, \forall n \in I\right\}$, the solution $x\left(n, n_{0}, \varphi\right)$ of (4) and (7) satisfies

$$
\Gamma_{2}<x\left(n, n_{0}, \varphi\right)<\Gamma_{1}, \forall n \in\left[n_{0}, \infty\right) .
$$

Proof. Set $x(n)=x\left(n, n_{0}, \varphi\right)$. Let $\left[n_{0}, T\right) \subseteq\left[n_{0}, \infty\right)$ be an interval such that

$$
x(n)>0, \forall n \in\left[n_{0}, T\right) .
$$

We claim that

$$
0<x(n)<\Gamma_{1}, \forall n \in\left[n_{0}, T\right) .
$$

For the sake of contradiction, we assume that (10) is not true. Then, one can find $n_{1} \in\left(n_{0}, T\right)$ such that

$$
x\left(n_{1}+1\right) \geq \Gamma_{1} \text { and } 0<x(n)<\Gamma_{1},
$$


for all $n \in\left[n_{0}-r, n_{1}+1\right)$. In view of (4), A.1, (11) and the fact that $\sup _{u \geq 0} u e^{-u}=\frac{1}{e}$, we have

$$
\begin{aligned}
0 \leq \Delta x\left(n_{1}\right)= & -\alpha\left(n_{1}\right) x\left(n_{1}+1\right) \\
+ & \beta\left(n_{1}\right) x\left(n_{1}-\tau\left(n_{1}\right)\right) e^{-\gamma\left(n_{1}\right) x\left(n_{1}-\tau\left(n_{1}\right)\right)} \\
& -H\left(n_{1}, x\left(n_{1}-\sigma\left(n_{1}\right)\right)\right) \\
\leq & -\alpha\left(n_{1}\right) x\left(n_{1}+1\right) \\
+ & \frac{\beta\left(n_{1}\right)}{\gamma\left(n_{1}\right)} \gamma\left(n_{1}\right) x\left(n_{1}-\tau\left(n_{1}\right)\right) e^{-\gamma\left(n_{1}\right) x\left(n_{1}-\tau\left(n_{1}\right)\right)} \\
\leq & -\alpha^{-} x\left(n_{1}+1\right)+\left(\frac{\beta}{\gamma}\right)^{+} \frac{1}{e} \\
\leq & \alpha^{-}\left[-\Gamma_{1}+\left(\frac{\beta}{\gamma}\right)^{+} \frac{1}{\alpha^{-}} e^{-}\right] \\
< & 0,
\end{aligned}
$$

which is a contradiction and this implies that (10) holds. Next, we show that

$$
x(n)>\Gamma_{2}, \forall n \in\left[n_{0}, T\right) .
$$

On the contrary, assume that there exists $n_{2} \in\left(n_{0}, T\right)$ such that

$$
x\left(n_{2}+1\right) \leq \Gamma_{2}, \text { and } x(n)>\Gamma_{2},
$$

for all $n \in\left[n_{0}-r, n_{2}+1\right)$. In virtue of A.1 and (10), we obtain

$$
\Gamma_{2}<x(n)<\Gamma_{1}, \quad \gamma^{+} x(n) \geq \gamma^{+} \frac{1}{\gamma} \geq 1
$$

for all $n \in\left[n_{0}-r, n_{2}+1\right)$. In view of (4), A.1, (13), (14) and the fact that $\min _{1 \leq u \leq \delta} u e^{-u}=\delta e^{-\delta}$, we have

$$
\begin{aligned}
0 \geq \Delta x\left(n_{2}\right) & =-\alpha\left(n_{2}\right) x\left(n_{2}+1\right) \\
& +\frac{\beta\left(n_{2}\right)}{\gamma\left(n_{2}\right)} \gamma\left(n_{2}\right) x\left(n_{2}-\tau\left(n_{2}\right)\right) e^{-\gamma\left(n_{2}\right) x\left(n_{2}-\tau\left(n_{2}\right)\right)} \\
& -H\left(n_{2}, x\left(n_{2}-\sigma\left(n_{2}\right)\right)\right) \\
& \geq-\alpha^{+} \Gamma_{2}+\frac{\beta^{-}}{\gamma^{+}} \gamma^{+} \Gamma_{1} e^{-\gamma^{+} \Gamma_{1}}-H^{+} \\
& \geq \alpha^{+}\left[-\Gamma_{2}+\frac{\beta^{-}}{\alpha^{+}} \Gamma_{1} e^{-\gamma^{+} \Gamma_{1}}-\frac{H^{+}}{\alpha^{+}}\right]>0,
\end{aligned}
$$

which is a contradiction and this implies that (12) holds. In view of (10) and (12), it follows that relation (8) is true. Hence the proof is complete.

\section{The Main Results}

Consider the linear difference system

$$
x(n+1)=A(n) x(n),
$$

where $x \in \mathbb{Z}^{m}$ and $A: \mathbb{Z} \rightarrow \mathbb{Z}^{m \times m}$ is a matrix sequence. In what follows, we denote by $\|\cdot\|$ any convenient norm either of a vector or of a matrix.
Definition 3.The difference system (15) is said to possess an exponential dichotomy on $\mathbb{Z}$ if there exist a projection $P$, that is, an $m \times m$ matrix $P$ such that $P^{2}=P$, and constants $K>0, v>0$ such that

$$
\left\|X(r) P X^{-1}(s+1)\right\| \leq K\left(\frac{1}{1+v}\right)^{r-s-1}, r \geq s
$$

and

$$
\left\|X(r)(I-P) X^{-1}(s+1)\right\| \leq K\left(\frac{1}{1+v}\right)^{s+1-r}, s \geq r
$$

where $X(t)$ is the fundamental solution matrix of (15) and $r, s \in \mathbb{Z}$.

Consider the following almost periodic difference system

$$
x(n+1)=A(n) x(n)+f(n, x),
$$

where $A: \mathbb{Z} \rightarrow \mathbb{Z}^{m \times m}$ is almost periodic matrix sequence and $f: \mathbb{Z} \times \mathbb{Z}^{m} \rightarrow \mathbb{Z}^{m}$ is almost periodic vector sequence.

Theorem 1.[23] If the linear system (15) admits exponential dichotomy, then system (16) has a bounded solution $x(n)$ in the form

$$
\begin{aligned}
x(n) & =\sum_{k=-\infty}^{n-1} X(n) P X^{-1}(k+1) f(k, x(k)) \\
& -\sum_{k=n}^{\infty} X(n)(I-P) X^{-1}(k+1) f(k, x(k)),
\end{aligned}
$$

where $X(n)$ is the fundamental solution matrix of (15).

The following result can be easily extracted from Lemma 2.15 in [25].

Theorem 2.Let $\alpha(n)>0$ be an almost periodic sequence on $\mathbb{Z}$ and

$$
\inf _{n \in \mathbb{Z}} \frac{\alpha(n)}{1+\alpha(n)}>0,
$$

then the linear system

$$
\Delta x(n)=-\alpha(n) x(n+1)
$$

admits an exponential dichotomy on $\mathbb{Z}$.

Set

$$
\mathscr{B}=\{\varphi: \varphi \text { is an almost periodic sequence on } \mathbb{Z}\} .
$$

If we define the norm $\|\varphi\|_{\mathscr{B}}=\sup _{n \in \mathbb{Z}}|\varphi(n)|$, for any $\varphi \in$ $\mathscr{B}$, then one can easily deduce that $\mathscr{B}$ is a Banach space.

We assume that

A. $2 \frac{\beta^{+}}{\alpha^{-}} \frac{1}{e^{2}}+\frac{L_{H}}{\alpha^{-}}<1$.

Theorem 3.Let A.1 and A.2 hold. Then, there exists a unique positive almost periodic solution of (4) in $\mathscr{B}^{*}:=\left\{\varphi: \varphi \in \mathscr{B}, \Gamma_{2}<\varphi(n)<\Gamma_{1}, \forall n \in \mathbb{Z}\right\}$. 
Proof.For any $\phi \in \mathscr{B}$, we consider an auxiliary equation

$$
\begin{aligned}
\Delta x(n) & =-\alpha(n) x(n+1)+\beta(n) \phi(n-\tau(n)) e^{-\gamma(n) \phi(n-\tau(n))} \\
& -H(n) \phi(n-\sigma(n)) .
\end{aligned}
$$

Since $\inf _{n \in \mathbb{Z}} \frac{\alpha(n)}{1+\alpha(n)}>0$, it follows from Theorem 2 that the linear system

$$
\Delta x(n)=-\alpha(n) x(n+1)
$$

admits an exponential dichotomy on $\mathbb{Z}$. By Theorem 1 and Theorem 2, we deduce that system (20) has a bounded solution of the form

$$
\begin{aligned}
x^{\phi}(n) & =\sum_{m=-\infty}^{n-1} \prod_{r=m}^{n-1}\left(\frac{1}{1+\alpha(r)}\right) \\
& \times\left[\beta(m) \phi(m-\tau(m)) e^{-\gamma(m) \phi(m-\tau(m))}\right. \\
& -H(m, \phi(m-\sigma(m)))] .
\end{aligned}
$$

By using H.1-H.2, the almost periodicity of $\prod_{r=m}^{n-1}\left(\frac{1}{1+\alpha(r)}\right)$ and the fact that the uniform limit of almost periodic sequences is also almost periodic, we deduce that $x^{\phi}$ is also almost periodic.

Define a mapping $T: \mathscr{B} \rightarrow \mathscr{B}$ by setting

$$
T(\phi(n))=x^{\phi}(n), \forall \phi \in \mathscr{B} .
$$

It is easy to see that $\mathscr{B}^{*}$ is a closed subset of $\mathscr{B}$. For any $\phi \in \mathscr{B}^{*}$, we have

$$
\begin{aligned}
x^{\phi}(n) & \leq \sum_{m=-\infty}^{n-1} \prod_{r=m}^{n-1}\left(\frac{1}{1+\alpha(r)}\right) \\
& \times \beta(m) \phi(m-\tau(m)) e^{-\gamma(m) \phi(m-\tau(m))} \\
& =\sum_{m=-\infty}^{n-1} \prod_{r=m}^{n-1}\left(\frac{1}{1+\alpha(r)}\right) \\
& \times \frac{\beta(m)}{\gamma(m)} \gamma(m) \phi(m-\tau(m)) e^{-\gamma(m) \phi(m-\tau(m))} .
\end{aligned}
$$

By the fact that $\sup _{u \geq 0} u e^{-u}=\frac{1}{e}$, we obtain

$x^{\phi}(n) \leq \sum_{m=-\infty}^{n-1} \prod_{r=m}^{n-1}\left(\frac{1}{1+\alpha^{-}}\right)\left(\frac{\beta}{\gamma}\right)^{+} \frac{1}{e}$.

Using that $\sum_{m=-\infty}^{n-1} \prod_{r=m}^{n-1}\left(\frac{1}{1+\alpha^{-}}\right)=\frac{1}{\alpha^{-}}$, we end up with

$$
x^{\phi}(n) \leq\left(\frac{\beta}{\gamma}\right)^{+} \frac{1}{\alpha^{-} e}<\Gamma_{1}, \forall n \in \mathbb{Z} .
$$

On the other hand, we have

$$
\begin{aligned}
x^{\phi}(n) & \geq \sum_{m=-\infty}^{n-1} \prod_{r=m}^{n-1}\left(\frac{1}{1+\alpha(r)}\right) \\
& \times\left[\frac{\beta(m)}{\gamma^{+}} \gamma^{+} \phi(m-\tau(m)) e^{-\gamma^{+} \phi(m-\tau(m))}-H^{+}\right] .
\end{aligned}
$$

By virtue of the fact that $\min _{1 \leq u \leq \kappa} u e^{-u}=\kappa e^{-\kappa}$, we obtain

$$
x^{\phi}(n) \geq \frac{\beta^{-}}{\alpha^{+}} \Gamma_{1} e^{-\gamma^{+} \Gamma_{1}}-\frac{H^{+}}{\alpha^{+}}>\Gamma_{2}, \forall n \in \mathbb{Z} .
$$

This tells that the mapping $T$ is a self-mapping from $\mathscr{B}^{*}$ to $\mathscr{B}^{*}$.

Next, we claim that $T$ is a contractive mapping on $\mathscr{B}^{*}$. Let $\varphi, \psi \in \mathscr{B}^{*}$. Then

$$
\begin{aligned}
& \|T(\varphi)-T(\psi)\|_{\mathscr{B}}=\sup _{n \in \mathbb{Z}}|T(\varphi(n))-T(\psi(n))| \\
= & \sup _{n \in \mathbb{Z}} \mid \sum_{m=-\infty}^{n-1} \prod_{r=m}^{n-1}\left(\frac{1}{1+\alpha(r)}\right) \beta(m)[\varphi(m-\tau(m)) \\
& \left.\left.e^{-\gamma(m) \varphi(m-\tau(m))}-\psi(m-\tau(m)) e^{-\gamma(m) \psi(m-\tau(m))}\right)\right] \\
- & H(m, \varphi(m-\sigma(m)))+H(m, \psi(m-\sigma(m))) \mid \\
= & \sup _{n \in \mathbb{Z}} \mid \sum_{m=-\infty}^{n-1} \prod_{r=m}^{n-1}\left(\frac{1}{1+\alpha(r)}\right) \frac{\beta(m)}{\gamma(m)}[\gamma(m) \varphi(m-\tau(m)) \\
& \left.\left.e^{-\gamma(m) \varphi(m-\tau(m))}-\gamma(m) \psi(m-\tau(m)) e^{-\gamma(m) \psi(m-\tau(m))}\right)\right] \\
- & H(m, \varphi(m-\sigma(m)))+H(m, \psi(m-\sigma(m))) \mid
\end{aligned}
$$

In virtue of the fact that $\sup _{u \geq 1}\left|\frac{1-u}{e^{u}}\right|=\frac{1}{e^{2}}$, we observe that

$$
\begin{aligned}
\left|x e^{-x}-y e^{-y}\right| & =\left|\frac{1-(x+\theta(y-x))}{e^{x+\theta(y-x)}}\right||x-y| \\
& \leq \frac{1}{e^{2}}|x-y|, x, y \in[1, \infty), 0<\theta<1 .
\end{aligned}
$$

By (8), we also observe

$$
\gamma(m) \varphi(m-\tau(m)) \geq \gamma(m) \Gamma_{2} \geq \gamma(m) \frac{1}{\gamma} \geq 1, \forall m \in \mathbb{Z} .
$$

Therefore, by (4), (5) and (25), we have

$$
\begin{aligned}
& \|T(\varphi)-T(\psi)\|_{\mathscr{B}} \leq \sup _{n \in \mathbb{Z}} \sum_{m=-\infty}^{n-1} \prod_{r=m}^{n-1}\left(\frac{1}{1+\alpha^{-}}\right) \\
\times & {\left[\frac{\beta(m)}{e^{2}}|\varphi(m-\tau(m))-\psi(m-\tau(m))|\right.} \\
+ & |H(m, \varphi(m-\sigma(m)))-H(m, \psi(m-\sigma(m)))|] \\
\leq & \left(\sup _{n \in \mathbb{Z}} \sum_{m=-\infty}^{n-1} \prod_{r=m}^{n-1}\left(\frac{1}{1+\alpha^{-}}\right)\right)\left(\frac{\beta^{+}}{e^{2}}+L_{H}\right)\|\varphi-\psi\| .
\end{aligned}
$$

Therefore, we end up with

$$
\|T(\varphi)-T(\psi)\|_{\mathscr{B}} \leq\left(\frac{\beta^{+}}{\alpha^{-} e^{2}}+\frac{L_{H}}{\alpha^{-}}\right)\|\varphi-\psi\|,
$$

which implies by (A.2) that the mapping $T$ is contractive on $\mathscr{B}^{*}$. Therefore, the mapping $T$ possesses a unique fixed point $\varphi^{*} \in \mathscr{B}^{*}$ such that $T \varphi^{*}=\varphi^{*}$. Thus, $\varphi^{*}$ is an almost periodic solution of (4) in the $\mathscr{B}^{*}$. The proof is complete. 
We assume that

$$
\text { A. } 3 \alpha^{-}>1+\frac{\beta^{+}}{e^{2}}+L_{H} \text {. }
$$

Theorem 4.Let A.1 and A.3 hold. Further, assume $x^{*}(n)$ is positive almost periodic solution of $(4)$ in the set $\mathscr{B}^{*}$. Then, the solution $x\left(n, n_{0}, \varphi\right)$ of $(4)$ with $\varphi \in C^{0}$ converges exponentially to $x^{*}(n)$ as $n \rightarrow \infty$.

Proof.Set $x(n)=x\left(n, n_{0}, \varphi\right)$ and $y(n)=x(n)-x^{*}(n)$, where $n \in\left[n_{0}-r, \infty\right)$. Then

$$
\begin{aligned}
\Delta y(n)= & -\alpha(n) y(n+1)+\beta(n)\left[x(n-\tau(n)) e^{-\gamma(n) x(n-\tau(n))}\right. \\
& \left.-x^{*}(n-\tau(n)) e^{-\gamma(n) x^{*}(n-\tau(n))}\right] \\
- & {\left[H(n, x(n-\sigma(n)))-H\left(n, x^{*}(n-\sigma(n))\right)\right] . }
\end{aligned}
$$

The result of Lemma 1 tells that $x(n)$ is positive and bounded on $\left[n_{0}, \infty\right)$ and

$$
\Gamma_{2}<x(n)<\Gamma_{1}, \forall n \in\left[n_{0}-r, \infty\right) .
$$

Define a function $\Phi(u)$ by setting

$$
\Phi(u)=e^{u}-\alpha^{-}+\beta^{+} \frac{1}{e^{2}} e^{u(r+1)}+L_{H} e^{u(r+1)}, u \in[0,1] .
$$

It is clear that $\Phi$ is continuous on $[0,1]$. Then, by (A.3) we have

$\Phi(0)=1-\alpha^{-}+\frac{\beta^{+}}{e^{2}}+L_{H}<0$,

which implies that there exist two constants $\eta>0$ and $0<$ $\lambda \leq 1$ such that

$$
\Phi(\lambda)=e^{\lambda}-\alpha^{-}+\beta^{+} \frac{1}{e^{2}} e^{\lambda(r+1)}+L_{H} e^{\lambda(r+1)}<-\eta<0 .
$$

We consider the discrete Lyapunov functional

$$
V(n)=|y(n)| e^{\lambda n} .
$$

Calculating the difference of $V(n)$ along the solution $y(n)$ of (26), we have

$$
\begin{aligned}
\Delta V(n) & =\Delta\left(|y(n)| e^{\lambda n}\right) \\
& =\Delta|y(n)| e^{\lambda(n+1)}+|y(n)| \Delta e^{\lambda n} \\
& \leq-\alpha(n)|y(n+1)| e^{\lambda(n+1)} \\
& +\beta(n) \mid x(n-\tau(n)) e^{-\gamma(n) x(n-\tau(n))} \\
& -x^{*}(n-\tau(n)) e^{-\gamma(n) x^{*}(n-\tau(n))} \mid e^{\lambda(n+1)} \\
& +\left|H(n, x(n-\sigma(n)))-H\left(n, x^{*}(n-\sigma(n))\right)\right| e^{\lambda(n+1)} \\
& +|y(n)|\left(e^{\lambda(n+1)}-e^{\lambda n}\right) \\
& \leq|y(n)| e^{\lambda(n+1)}-\alpha(n)|y(n+1)| e^{\lambda(n+1)} \\
& +\left[\beta(n) \mid x(n-\tau(n)) e^{-\gamma(n) x(n-\tau(n))}\right. \\
& -x^{*}(n-\tau(n)) e^{-\gamma(n) x^{*}(n-\tau(n))} \mid \\
& \left.+\left|H(n, x(n-\sigma(n)))-H\left(n, x^{*}(n-\sigma(n))\right)\right|\right] e^{\lambda(n+1)}
\end{aligned}
$$

for all $n \geq n_{0}$.

Let

$$
M:=e^{\lambda n_{0}}\left(\max _{n \in\left[n_{0}, \infty\right)}\left|\varphi(n)-x^{*}(n)\right|+1\right), \forall n \geq n_{0} .
$$

Then, we claim that

$$
V(n)=|y(n)| e^{\lambda n}<M, \forall n \geq n_{0} .
$$

Assume, on the contrarily, that there exists $n_{*}>n_{0}$ such that

$$
V\left(n_{*}\right) \geq M \text { and } V(n)<M, \forall n \in\left[n_{0}-r, n_{*}\right),
$$

which implies that

$$
V\left(n_{*}\right)-M \geq 0 \text { and } V(n)-M<0, \forall n \in\left[n_{0}-r, n_{*}\right) .
$$

In virtue of (25), (31) and (32), we obtain

$$
\begin{aligned}
0 & \leq \Delta\left(V\left(n_{*}\right)-M\right)=\Delta V\left(n_{*}\right) \\
& \leq\left|y\left(n_{*}\right)\right| e^{\lambda\left(n_{*}+1\right)}-\alpha\left(n_{*}\right)\left|y\left(n_{*}+1\right)\right| e^{\lambda\left(n_{*}+1\right)} \\
& +\left[\beta\left(n_{*}\right) \mid x\left(n_{*}-\tau\left(n_{*}\right)\right) e^{-\gamma\left(n_{*}\right) x\left(n_{*}-\tau\left(n_{*}\right)\right)}\right. \\
& -x^{*}\left(n_{*}-\tau\left(n_{1}\right)\right) e^{-\gamma\left(n_{*}\right) x^{*}\left(n_{*}-\tau\left(n_{*}\right)\right)} \mid \\
& \left.+\left|H\left(n_{*}, x\left(n_{*}-\sigma(n)\right)\right)-H\left(n_{*}, x^{*}\left(n_{*}-\sigma(n)\right)\right)\right|\right] e^{\lambda\left(n_{*}+1\right)} \\
& \leq\left|y\left(n_{*}\right)\right| e^{\lambda\left(n_{*}+1\right)}-\alpha\left(n_{*}\right)\left|y\left(n_{*}+1\right)\right| e^{\lambda\left(n_{*}+1\right)} \\
& +\frac{\beta\left(n_{*}\right)}{e^{2}}\left|y\left(n_{*}-\tau\left(n_{*}\right)\right)\right| e^{\lambda\left(n_{*}+1-\tau\left(n_{*}\right)\right)} e^{\lambda \tau\left(n_{*}\right)} \\
& +L_{H}\left|y\left(n_{*}-\sigma\left(n_{*}\right)\right)\right| e^{\lambda\left(n_{*}+1-\sigma\left(n_{*}\right)\right)} e^{\lambda \sigma\left(n_{*}\right)} \\
& \leq\left(e^{\lambda}-\alpha^{-}\right) M+\frac{\beta^{+}}{e^{2}} M e^{\lambda(r+1)}+L_{H} M e^{\lambda(r+1)} \\
& =\left[e^{\lambda}-\alpha^{-}+\frac{\beta^{+}}{e^{2}} e^{\lambda(r+1)}+L_{H} e^{\lambda(r+1)}\right] M .
\end{aligned}
$$

Thus,

$$
e^{\lambda}-\alpha^{-}+\frac{\beta^{+}}{e^{2}} e^{\lambda(r+1)}+L_{H} e^{\lambda(r+1)} \geq 0
$$

which contradicts (29). Hence (31) holds. It follows that $|y(n)|<M e^{-\lambda n}$ for all $n \geq n_{0}$. The proof is complete.

In what follows, we construct an example whose aim is not standing at generality but illustrating how the main theorems can be used.

Example 1.Consider the following Nicholson's blowflies model with a nonlinear harvesting term of the form

$$
\begin{aligned}
\Delta x(n) & =-\left(18+\cos ^{2} n\right) x(n+1) \\
& +e^{e-1}(20+0.01|\sin \sqrt{5} n|) x\left(n-e^{2|\cos \sqrt{3} n|}\right) \\
& \times e^{-x\left(n-e^{2|\cos \sqrt{3} n|}\right)} \\
& -\frac{\sqrt{5}}{14}|\sin n+\cos \sqrt{11} n| \frac{x^{2}\left(n-e^{2|\cos \sqrt{3} n|}\right)}{1+x^{2}\left(n-e^{2|\cos \sqrt{3} n|}\right)},
\end{aligned}
$$


where

$$
\begin{gathered}
\alpha(n)=18+\cos ^{2} n, \beta(n)=e^{e-1}(20+0.01|\sin \sqrt{5} n|), \\
\tau(n)=e^{2|\cos \sqrt{3} n|}, \gamma(n)=1
\end{gathered}
$$

and

$H(n, x)=\frac{\sqrt{5}}{14}|\sin n+\cos \sqrt{11} n| \frac{x^{2}(n)}{1+x^{2}(n)}, \sigma(n)=e^{2|\cos \sqrt{3} n|}$.

It is clear that

$$
\begin{aligned}
& \alpha^{-}=18, \alpha^{+}=19, \beta^{-}=20 e^{e-1}, \beta^{+}=20.01 e^{e-1}, \\
& \gamma^{+}=\gamma^{-}=1 \text { and } H^{+}=0.32
\end{aligned}
$$

It is not difficult to show that $H$ satisfies Lipshitz condition with $L_{H}=0.32$. Thus, conditions H.1-H.2 hold. Let $\Gamma_{1}=e$ and $\Gamma_{2}=1$. Then

$$
\begin{gathered}
\Gamma_{1}=e>\Gamma_{2}=1,\left(\frac{\beta}{\gamma}\right)^{+} \frac{1}{\alpha^{-} e} \approx 2.28<e, \\
\frac{\beta^{-}}{\alpha^{+}} \Gamma_{1} e^{-\gamma^{+} \Gamma_{1}}-\frac{H^{+} \Gamma_{1}}{\alpha^{+}} \approx 1.00685>1
\end{gathered}
$$

and this shows that condition A. 1 is satisfied. It remains to check conditions A.2 and A.3. Indeed, one can see the validity of these conditions since

$\frac{\beta^{+}}{\alpha^{-}} \frac{1}{e^{2}}+\frac{L_{H}}{\alpha^{-}} \approx 0.85<1$ and $1+\frac{\beta^{+}}{e^{2}}+L_{H} \approx 16.42<18$.

Therefore, we conclude that all assumptions of Theorem 3 and Theorem 4 are fulfilled. Hence, system (34) has a positive almost periodic solution $x^{*}$ in $\mathscr{B}^{*}=\{\varphi: \varphi \in \mathscr{B}$, $1<\varphi(n)<e, \forall n \in \mathbb{Z}\}$. Moreover, if $\varphi \in C^{0}$, then $x\left(n, n_{0}, \varphi\right)$ converges exponentially to $x^{*}$ as $n \rightarrow \infty$.

\section{Some Remarks}

1.Due to certain biological restrictions, it is agreed that the solutions under study in this paper are positive. This is justified by the choice of the initial condition in form of (7). Indeed, one can easily show that the solutions of (4) with (7) remain positive for all $n \in \mathbb{Z}$.

2.It is worth mentioning here that most of the discrete Nicholson's models investigated in the literature have involved a linear part of form $\Delta x(n)=-\alpha(n) x(n)$. In this paper, however, we consider Nicholson's model of form (4) to guarantee the convergence of the series appearing in the solution representation (22).

3.As Nicholson's model under consideration is involving a nonlinear harvesting term, one can easily figure out that some of the results reported in the literature might be no longer applicable for proving the existence and exponential convergence of almost periodic solutions of (4). This implies that the main theorems of this paper improve as well as extend some of previously obtained results among them we list for instance $[4,9,10,23]$.

4.The results of this paper could be generalized to Nicholson's model with patch structure and nonlinear harvesting terms.

\section{References}

[1] W. S. Gurney, S. P. Blythe, R. M. Nisbet: Nicholsons blowflies (revisited), Nature, 287, 17-21 (1980).

[2] A. J. Nicholson: An outline of the dynamics of animal populations, Austral. J. Zool., 2 , 9-25 (1954).

[3] V. L. Kocic, G. Ladas: Oscillation and global attractivity in the discrete model of Nicholsons blowflies, Appl. Anal., 38, 21-31 (1990).

[4] M. R. S. Kulenovic, G. Ladas, Y. S. Sficas: Global attractivity in Nicholsons blowflies, Comput. Math. Appl., 18, 925-928 (1989).

[5] J. Li, C. Du: Existence of positive periodic solutions for a generalized Nicholson's blowflies model, J. Comput. Appl. Math., 221, 226-233 (2008).

[6] W. Li, Y. Fan: Existence and global attractivity of positive periodic solutions for the impulsive delay Nicholson's blowflies model, J. Comput. Appl. Math., 201, 55-68 (2007).

[7] J. Wei, M. Y. Li: Hopf bifurcation analysis in a delayed Nicholson blowflies equation, Nonlinear Anal., 60, 13511367 (2005).

[8] S. H. Saker, S. Agarwal: Oscillation and global attractivity in a periodic Nicholson's blowflies model, Math. Comput. Modelling, 35 , 719-731 (2002).

[9] B. G. Zhang, H. X. Xu: A note on the global attractivity of a discrete model of Nicholson's blowflies, Discrete Dyn. Nat. Soc., 3, 51-55 (1999).

[10] J. W. H. So , J. S. Yu: On the stability and uniform persistence of a discrete model of Nicholson's blowflies, J. Math. Anal. Appl., 193, 233-244 (1995).

[11] J. O. Alzabut: Almost periodic solutions for impulsive delay Nicholson's blowflies population model, J. Comput. Appl. Math., 234, 233-239 (2010).

[12] L. Berezansky, E. Braverman, L. Idels: Nicholson's blowflies differential equations revisited: Main results and open problems, Appl. Math. Modelling, 34, 1405-1417 (2010).

[13] F. Long: Postive almost periodic solution for a class of Nicholson's blowflies model with a linear harvesting term, Nonlinear Anal., 13, 686-693 (2012).

[14] F. Long, M. Yang: Positive periodic solutions of delayed Nicholsons blowflies model with a linear harvesting term, Electron. J. Qual. Theory Differ. Equ., 41, 1-11 (2011).

[15] W. Zhao, C. Zhu, H. Zhu: On positive solution for the delay Nicholson's blowflies model with a harvasting term, Appl. Math. Modelling, 36, 3335-3340 (2012).

[16] X. Liu, J. Meng: The positive almost periodic solution for Nicholson-type delay systems with linear harvesting terms, Appl. Math. Modelling, 36, 3289-3298 (2012).

[17] Q. Zhou: The positive periodic solution for Nicholsontype delay system with linear harvesting terms, Appl. Math. Modelling, 37, 5581-5590 (2013).

[18] L. Wang: Almost periodic solution for Nicholsons blowflies model with patch structure and linear harvesting terms, Appl. Math. Modelling, 37, 2153-2165 (2013).

[19] P. Amster, A. Deboli: Existence of positive T-periodic solutions of a generalized Nicholson's blowflies model with a nonlinear harvesting term, Applied Mathematics Letters, 25, 1203-1207 (2012). 
[20] S. Goldberg: Introduction to Difference Equations: With Illustrative Examples from Economics, Psychology, and Sociology, Dover Publications, (1986).

[21] S. N. Elaydi: An Introduction to Difference Equations, Springer, (2005).

[22] R. P. Agarwal: Difference Equations and Inequalities: Theory, methods, and applications, Monographs and Textbooks in Pure and Applied Mathematics, Second ed., Marcel Dekker Inc., New York, 228, (2000).

[23] J. O. Alzabut, Y. Bolat, T. Abdeljawad: Almost periodic dynamics of a discrete Nicholsons blowflies model involving a linear harvesting term, Adv. Difference Equ., (2012).

[24] D. Cheban, C. Mammana: Invariant manifolds, global attractors and almost periodic solutions of nonautonomous difference equations, Nonlinear Anal., 56, 465-484 (2004).

[25] Y. Li, C. Wang: Almost periodic functions on time scales and applications, Discrete Dyn. Nat. Soc., 2011.

[26] J. W. H. So, Y. Yang, Dirichlet problem for the diffusive Nicholson's blowflies equation, J. Differential Equations, 150, 317-348 (1998).

[27] D. M. Thomas, F. Robbins, Analysis of a nonautonomous Nicholson blowfly model, Phys., A 273, 1-2, 198-211 (1999).

[28] J. W. H. So, J. Wu, Y. Yang, Numerical steady state and Hopf bifurcation analysis on the diffusive Nicholson's blowflies equation, Appl. Math. Comput., 111, 53-69 (2000).

[29] S. H. Saker, Oscillation of continuous and discrete diffusive delay Nicholsons blowflies models, Appl. Math. Comput., 167, 179-197 (2005) .

[30] S. H. Saker, B. G. Zhang, Oscillation in a discrete partial delay Nicholson's Blowflies Model, Math. Computer Modelling, 36, 9-10, 1021-1026 (2002).

[31] S. H. Saker, Oscillation of continuous and discrete diffusive delay Nicholsons blowflies models, Appl. Math. Comput., 167, 179-197 (2005)

[32] T. Yi, X. Zou, Global attractivity of the diffusive Nicholson blowflies equation with Neumann boundary condition: A non-monotone case, J. Differential Equations, 245, 33763388 (2008). 